\title{
U-Type Designs via New Generalized Partially Balanced Incomplete Block Designs with $m=4,5$ and 7 Associated Classes
}

\author{
Imane Rezgui ${ }^{1}$, Zebida Gheribi-Aoulmi ${ }^{1}$, Hervé Monod ${ }^{2}$ \\ ${ }^{1}$ Department of Mathematics, University of Constantine 1, Constantine, Algeria \\ ${ }^{2}$ INRA, UR MalAGE, Jouy-en-Josas, Paris, France \\ Email: rezgui imane@yahoo.fr, gheribiz@yahoo.fr, herve.monod@jouy.inra.fr
}

Received 10 January 2015; accepted 28 January 2015; published 4 February 2015

Copyright (C) 2015 by authors and Scientific Research Publishing Inc.

This work is licensed under the Creative Commons Attribution International License (CC BY).

http://creativecommons.org/licenses/by/4.0/

(c) $\underset{\mathrm{EY}}{0}$ Open Access

\section{Abstract}

The traditional combinatorial designs can be used as basic designs for constructing designs of computer experiments which have been used successfully till now in various domains such as engineering, pharmaceutical industry, etc. In this paper, a new series of generalized partially balanced incomplete blocks PBIB designs with $m$ associated classes $(m=4,5$ and 7$)$ based on new generalized association schemes with number of treatments $v$ arranged in $w$ arrays of $n$ rows and $l$ columns $(w \geq 2, n \geq 2, l \geq 2)$ is defined. Some construction methods of these new PBIB are given and their parameters are specified using the Combinatory Method ( $s$ ). For $n$ or $l$ even and $s$ divisor of $\boldsymbol{n}$ or $l$, the obtained PBIB designs are resolvable PBIB designs. So the Fang RBIBD method is applied to obtain a series of particular $U$-type designs $U\left(w n l ; \frac{w n l^{r}}{2 s}\right)$ ( $r$ is the repetition number of each treatment in our resolvable PBIB design).

\section{Keywords}

Association Scheme, Combinatory Method (s), Resolvable Partially Balanced Incomplete Block Design, $U$-Type Design

\section{Introduction}

Designs of computer experiments drew a wide attention in the previous two decades and were still being used

How to cite this paper: Rezgui, I., Gheribi-Aoulmi, Z. and Monod, H. (2015) U-Type Designs via New Generalized Partially Balanced Incomplete Block Designs with $m=4,5$ and 7 Associated Classes. Applied Mathematics, 6, 242-264. 
successfully till now in various domains. Among the various construction methods of these designs, the traditional combinatorial designs can be used as basic designs (example: [1] [2]) and particularly the PBIB designs. The association schemes of two or three associated classes have been widely studied, while it is not the case of those over three associated classes. However, some association schemes with five associated classes have been studied (example: [3]). Besides, a method to obtain new association schemes by crossing or nesting other association schemes was given by Bailey [4] using the character tables and strata of the initial association schemes to find the parameters of the obtained association schemes.

In this paper, new association schemes with 4, 5 and 7 associated classes are described starting by a geometric representation. The parameter expressions of these association schemes are given. Moreover, some methods to construct the PBIB designs based on these association schemes are explained using an accessible construction method called the Combinatory Method (s) [5], which allows obtaining a series of PBIB designs by only using these association schemes. The parameters expressions of these new designs are given. In addition, for $n$ or $l$ even and $s$ divisor of $n$ or $l$, the obtained PBIB are resolvable PBIB. Then, a series of $U$-type designs $U\left(w n l ; \frac{w n l}{2 s}\right)$ ( $r$ is the repetition number of each treatment in the resolvable PBIB design) is obtained by applying the RBIBD method [6] on these designs.

The paper is organized as follows. In Section 2, we give new definitions of generalized association schemes with $m(=4,5$ and 7$)$ associated classes, starting by geometric representation and we give their parameters as properties. Section 3 describes a series of construction method using the Combinatory Method (s) for obtaining the PBIB designs associated to our generalized association schemes. We give the series of the $U$-type designs associated to our constructed PBIB designs in Section 4. We achieve our paper with a Conclusion.

Recall some definitions:

Definition 1. An m-association scheme $(m \geq 2)$ of $v$ treatments [7] is a relation satisfying the following conditions:

1) Any two treatments are either $1^{\text {st }}, 2^{\text {nd }}, \cdots$, or $m^{\text {th }}$ associates. The relation of association is symmetric, i.e., if the treatment $\alpha$ is an $i^{\text {th }}$ associate of $\beta$, then $\beta$ is an $i^{\text {th }}$ associate of $\alpha(i=1,2, \cdots m)$.

2) Each treatment $\alpha$ has $n_{i} i^{\text {th }}$ associates, the number $n_{i}$ being independent of $\alpha(i=1,2, \cdots m)$.

3) If any two treatments $\alpha$ and $\beta$ are $i^{\text {th }}$ associates, then the number of treatments that are $j^{\text {th }}$ associates of $\alpha$ and $k^{\text {th }}$ associates of $\beta$ is $p_{j k}^{i}$ and is independent of the pair of $i^{\text {th }}$ associates $\alpha$ and $\beta(i, j, k=1,2, \cdots m)$.

The numbers $v, n_{i}(i=1,2, \cdots m)$ and $p_{j k}^{i}(i, j, k=1,2, \cdots m)$ are called the parameters of the association scheme.

Definition 2. A PBIB design [7], based on an m-association scheme $(m \geq 2)$, with parameters $v, b, r, k, \lambda_{i}$, $i=1,2, \cdots m$, is a block design with $v$ treatments and $b$ blocks of size $k$ each such that every treatment occurs in $r$ blocks and any two distinct treatments being $i^{\text {th }}$ associate occur together in exactly $\lambda_{i}$ blocks. The number $\lambda_{i}$ is independent of the pair of $i^{\text {th }}$ associates $(i=1,2, \cdots m)$.

A parallel class of PBIB is a collection of disjoint blocks from the $b$ blocks whose union is $V$. A partition of the $b$ blocks into $q=b / r$ parallel classes is called a resolution, and PBIB design is resolvable if it has at least one resolution and it denoted by RPBIB design.

The Combinatory Method (s) [5]:

Let an array of $n$ rows and $l$ columns as follows:

\begin{tabular}{cccccc}
\hline$a_{11}$ & $a_{12}$ & $\ldots$ & $a_{1 j}$ & $\ldots$ & $a_{1 l}$ \\
$a_{21}$ & $a_{22}$ & $\ldots$ & $a_{2 j}$ & $\ldots$ & $a_{2 l}$ \\
$\vdots$ & $\vdots$ & $\vdots$ & $\vdots$ & $\vdots$ & $\vdots$ \\
$a_{i 1}$ & $a_{i 2}$ & $\ldots$ & $a_{i j}$ & $\ldots$ & $a_{i l}$ \\
$\vdots$ & $\vdots$ & $\vdots$ & $\vdots$ & $\vdots$ & $\vdots$ \\
$a_{n 1}$ & $a_{n 2}$ & $\ldots$ & $a_{n j}$ & $\ldots$ & $a_{n l}$ \\
\hline
\end{tabular}


Consider $s$ different elements of the same row $i \quad(2 \leq s<l)$, and associate with them $s$ other elements of a row $i^{\prime} \quad\left(i^{\prime} \neq i\right)$, respecting the correspondence between the elements $a_{i j}$ and $a_{i^{\prime}}$. Bringing together the $2 s$ elements in the same block and making all possible combinations, we obtain a partially balanced incomplete block design of size $k=2 s$.

Definition 3. Let $U\left(v ; q_{1}, \cdots, q_{r}\right)$ denote a design of $v$ runs and $r$ factors with respective $q_{1}, \cdots, q_{r}$ levels. This design corresponds to an $v \times r$ matrix $X=\left(x^{1}, \cdots, x^{r}\right)$ such that the $i^{\text {th }}$ column $x^{i}$ takes values from a set of $q_{i}$ elements, say $\left\{1, \cdots, q_{i}\right\}$, equally often. The set of all such designs, called U-type designs in the statistical literature, is denoted by $U\left(v ; q_{1}, \cdots, q_{r}\right)$. Obviously, $v$ must be a multiple of $q_{i}(1 \leq i \leq r)$. When all the $q_{i}$ $(1 \leq i \leq r)$ are equal to $q$, we denote it by $U\left(v ; q^{r}\right)$. Note that the rows and columns of $X$ are identified with the runs and factors respectively [6].

\section{Generalized Rectangular Right Angular Association Schemes ( $m$ ) ( $m=4,5$ and 7 Associated Classes)}

\subsection{Generalized Rectangular Right Angular Association Scheme (4)}

Let $V$ be a set of $v=w n l$ treatments, $(w \geq 2, n \geq 2, l \geq 2)$, to which we associate a geometrical representation in the following way:

Each treatment of $V$ is associated with a unique triplet of the set $A^{1} \cup \cdots \cup A^{g} \cup \cdots \cup A^{w}$ where:

$$
A^{g}=\left\{(x, y, z) \in \mathbb{N}^{3} / 1 \leq x \leq l, 1 \leq y \leq n, z=g\right\}, \quad g=1, \cdots, w
$$

Let $\alpha$ be a treatment of coordinates $(x, y, z) \in A^{g}$

- $A_{1}^{g}=\left\{\left(x^{\prime}, y^{\prime}, z^{\prime}\right) \in A^{g} / x^{\prime} \neq x, y^{\prime}=y\right\}$ corresponds to the treatments $1^{\text {st }}$ associated to $\alpha$

- $A_{2}^{g}=\left\{\left(x^{\prime}, y^{\prime}, z^{\prime}\right) \in A^{g} / x^{\prime}=x, y^{\prime} \neq y\right\}$ corresponds to the treatments $2^{\text {nd }}$ associated to $\alpha$.

- $A_{3}^{g}=\left\{\left(x^{\prime}, y^{\prime}, z^{\prime}\right) \in A^{g} / x^{\prime} \neq x, y^{\prime} \neq y\right\}$ corresponds to the treatments $3^{\text {rd }}$ associated to $\alpha$.

- $A_{4}^{g}=\left\{\left(x^{\prime}, y^{\prime}, z^{\prime}\right) \in A^{g^{\prime}}, g^{\prime} \neq g\right\}$ corresponds to the treatments $4^{\text {th }}$ associated to $\alpha$.

This geometric representation describes a new association scheme, we call it for convenience, generalized rectangular right angular association scheme (4) with four associated classes, to which we give the following equivalent definition:

Definition 4. A generalized rectangular right angular association scheme (4) is an arrangement of $v=w n l(w \geq$ $2, n \geq 2, l \geq 2$ ) treatments in $w$ arrays of $n$ rows and l columns such that, with respect to each treatment $\alpha$ :

1) The first associates of $\alpha$ are the other treatments of the same row in the same array.

2) The second associates of $\alpha$ are the other treatments of the same column in the same array.

3) The third associates of $\alpha$ are the remaining treatments in the same array.

4) The fourth associates of $\alpha$ are the other treatments of the other arrays.

Property 1. The parameters of generalized rectangular right angular association schemes (4) are:

$$
\begin{aligned}
v=w n l, n_{1}=l-1, & n_{2}=n-1, n_{3}=(n-1)(l-1), n_{4}=(w-1) n l \\
P_{1} & =\left(\begin{array}{cccc}
l-2 & 0 & 0 & 0 \\
0 & 0 & n-1 & 0 \\
0 & n-1 & (l-2)(n-1) & 0 \\
0 & 0 & 0 & (w-1) \ln
\end{array}\right) \\
P_{2} & =\left(\begin{array}{cccc}
0 & 0 & l-1 & 0 \\
0 & n-2 & 0 & 0 \\
l-1 & 0 & (l-1)(n-2) & 0 \\
0 & 0 & 0 & (w-1) \ln
\end{array}\right)
\end{aligned}
$$




$$
\begin{aligned}
P_{3} & =\left(\begin{array}{cccc}
0 & 1 & l-2 & 0 \\
1 & 0 & n-2 & 0 \\
l-2 & n-2 & (l-2)(n-2) & 0 \\
0 & 0 & 0 & (w-1) \ln
\end{array}\right) \\
P_{4} & =\left(\begin{array}{cccc}
0 & 0 & 0 & l-1 \\
0 & 0 & 0 & n-1 \\
0 & 0 & 0 & (l-1)(n-1) \\
l-1 & n-1 & (l-1)(n-1) & (w-2) \ln
\end{array}\right)
\end{aligned}
$$

Definition 5. A PBIB design based on a generalized rectangular right angular association scheme (4) is called generalized rectangular right angular $\mathrm{GPBIB}_{4}$ design.

\subsection{Generalized Rectangular Right Angular Association Scheme}

Let $V$ be a set of $v=w n l$ treatments, ( $w \geq 2, n \geq 2, l \geq 2$ ), to which we associate a geometrical representation in the following way:

Each treatment of $V$ is associated with a unique triplet (of coordinates) of the set $A^{1} \cup \cdots \cup A^{g} \cup \cdots \cup A^{w}$ where:

$$
A^{g}=\left\{(x, y, z) \in \mathbb{N}^{3} / 1 \leq x \leq l, 1 \leq y \leq n, z=g\right\}, \quad g=1, \cdots, w
$$

Let $\alpha$ be a treatment of coordinates $(x, y, z) \in A^{g}$

- $A_{1}^{g}=\left\{\left(x^{\prime}, y^{\prime}, z^{\prime}\right) \in A^{g} / x^{\prime} \neq x, y^{\prime}=y\right\}$ corresponds to the treatments $1^{\text {st }}$ associated to $\alpha$

- $A_{2}^{g}=\left\{\left(x^{\prime}, y^{\prime}, z^{\prime}\right) \in A^{g} / x^{\prime}=x, y^{\prime} \neq y\right\}$ corresponds to the treatments $2^{\text {nd }}$ associated to $\alpha$.

- $A_{3}^{g}=\left\{\left(x^{\prime}, y^{\prime}, z^{\prime}\right) \in A^{g} / x^{\prime} \neq x, y^{\prime} \neq y\right\}$ corresponds to the treatments $3^{\text {rd }}$ associated to $\alpha$.

- $A_{4}^{g}=\left\{\left(x^{\prime}, y^{\prime}, z^{\prime}\right) \in A^{g^{\prime}}, g^{\prime} \neq g / y^{\prime}=y\right\}$ corresponds to the treatments $4^{\text {th }}$ associated to $\alpha$.

- $A_{5}^{g}=\left\{\left(x^{\prime}, y^{\prime}, z^{\prime}\right) \in A^{g^{\prime}}, g^{\prime} \neq g / y^{\prime} \neq y\right\}$ corresponds to the treatments $5^{\text {th }}$ associated to $\alpha$.

This geometric representation describes a new association scheme, we call it for convenience, generalized rectangular right angular association scheme (5) with five associated classes, to which we give the following equivalent definition:

Definition 6. A generalized rectangular right angular association scheme (5) is an arrangement of $v=w n l(w$ $\geq 2, n \geq 2, l \geq 2)$ treatments in $w(n \times l)$ rectangular arrays such that, with respect to each treatment $\alpha$ :

1) The first associates of $\alpha$ are the other treatments of the same row in the same array.

2) The second associates of $\alpha$ are the other treatments of the same column in the same array.

3) The third associates of $\alpha$ are the remaining treatments in the same array.

4) The fourth associates of $\alpha$ are the treatments of same row as $\alpha$, of the other arrays.

5) The fifth associates of $\alpha$ are the remaining treatments in the other arrays.

Property 2. The parameters of the generalized rectangular right angular association schemes (5) are:

$$
\begin{aligned}
& v=w n l, n_{1}=l-1, \quad n_{2}=n-1, \quad n_{3}=(n-1)(l-1), \quad n_{4}=(w-1) l, \quad n_{5}=(w-1)(n-1) l \\
& P_{1}=\left(\begin{array}{ccccc}
l-2 & 0 & 0 & 0 & 0 \\
0 & 0 & n-1 & 0 & 0 \\
0 & n-1 & (l-2)(n-1) & 0 & 0 \\
0 & 0 & 0 & l & 0 \\
0 & 0 & 0 & 0 & (w-1)(n-1) l
\end{array}\right)
\end{aligned}
$$




$$
\begin{aligned}
& P_{2}=\left(\begin{array}{ccccc}
0 & 0 & l-1 & 0 & 0 \\
0 & n-2 & 0 & 0 & 0 \\
l-1 & 0 & (l-1)(n-2) & 0 & 0 \\
0 & 0 & 0 & 0 & l \\
0 & 0 & 0 & l & (w-1)(n-2) l
\end{array}\right) \\
& P_{3}=\left(\begin{array}{ccccc}
0 & 1 & l-2 & 0 & 0 \\
1 & 0 & n-2 & 0 & 0 \\
l-2 & n-2 & (l-2)(n-2) & 0 & 0 \\
0 & 0 & 0 & 0 & l \\
0 & 0 & 0 & l & (w-2)(n-2) l
\end{array}\right) \\
& P_{4}=\left(\begin{array}{ccccc}
0 & 0 & 0 & l-1 & 0 \\
0 & 0 & 0 & 0 & n-1 \\
0 & 0 & 0 & 0 & (l-1)(n-1) \\
l-1 & 0 & 0 & (w-2) l & 0 \\
0 & n-1 & (l-1)(n-1) & 0 & (w-2)(l-1)(n-1)
\end{array}\right) \\
& P_{5}=\left(\begin{array}{ccccc}
0 & 0 & 0 & 0 & l-1 \\
0 & 0 & 0 & 1 & n-2 \\
0 & 0 & 0 & l-1 & (l-1)(n-2) \\
0 & 1 & l-1 & 0 & (w-2) l \\
l-1 & n-2 & (l-1)(n-2) & (w-2) l & (w-2)(n-2) l
\end{array}\right)
\end{aligned}
$$

Definition 7. A PBIB design based on a generalized rectangular right angular association scheme (5) is called generalized rectangular right angular $\mathrm{GPBIB}_{5}$ design.

\subsection{Generalized Rectangular Right Angular Association Scheme (7)}

Let $V$ be a set of $v=w n l$ treatments, ( $w \geq 2, n \geq 2, l \geq 2)$, to which we associate a geometrical representation in the following way:

Each treatment of $V$ is associated with a unique triplet of the set $A^{1} \cup \cdots \cup A^{g} \cup \cdots \cup A^{w}$ where:

$$
A^{g}=\left\{(x, y, z) \in \mathbb{N}^{3} / 1 \leq x \leq l, 1 \leq y \leq n, z=g\right\}, \quad g=1, \cdots, w
$$

Let $\alpha$ be a treatment of coordinates $(x, y, z) \in A^{g}$

- $A_{1}^{g}=\left\{\left(x^{\prime}, y^{\prime}, z^{\prime}\right) \in A^{g} / x^{\prime} \neq x, y^{\prime}=y\right\} \quad$ corresponds to the treatments $1^{\text {st }}$ associated to $\alpha$

- $A_{2}^{g}=\left\{\left(x^{\prime}, y^{\prime}, z^{\prime}\right) \in A^{g} / x^{\prime}=x, y^{\prime} \neq y\right\}$ corresponds to the treatments $2^{\text {nd }}$ associated to $\alpha$.

- $A_{3}^{g}=\left\{\left(x^{\prime}, y^{\prime}, z^{\prime}\right) \in A^{g} / x^{\prime} \neq x, y^{\prime} \neq y\right\} \quad$ corresponds to the treatments $3^{\text {th }}$ associated to $\alpha$.

- $A_{4}^{g}=\left\{\left(x^{\prime}, y^{\prime}, z^{\prime}\right) \in A^{g^{\prime}}, g^{\prime} \neq g / x^{\prime}=x, y^{\prime}=y\right\}$ corresponds to the treatments $4^{\text {th }}$ associated to $\alpha$.

- $A_{5}^{g}=\left\{\left(x^{\prime}, y^{\prime}, z^{\prime}\right) \in A^{g^{\prime}}, g^{\prime} \neq g / x^{\prime} \neq x, y^{\prime}=y\right\}$ corresponds to the treatments $5^{\text {th }}$ associated to $\alpha$.

- $A_{6}^{g}=\left\{\left(x^{\prime}, y^{\prime}, z^{\prime}\right) \in A^{g^{\prime}}, g^{\prime} \neq g / x^{\prime}=x, y^{\prime} \neq y\right\}$ corresponds to the treatments $6^{\text {th }}$ associated to $\alpha$.

- $A_{7}^{g}=\left\{\left(x^{\prime}, y^{\prime}, z^{\prime}\right) \in A^{g^{\prime}}, g^{\prime} \neq g / x^{\prime} \neq x, y^{\prime} \neq y\right\}$ corresponds to the treatments $7^{\text {th }}$ associated to $\alpha$.

This geometric representation describes a new association scheme, we call it for convenience, generalized rectangular right angular association scheme (7) with seven associated classes, to which we give the following equivalent definition:

Definition 8. A generalized rectangular right angular association scheme (7) is an arrangement of $v=w n l$ 
$(w \geq 2, n \geq 2, l \geq 2)$ treatments in $w$ arrays of $n$ rows and $l$ columns such that, with respect to each treatment $\alpha$ :

1) The first associates of $\alpha$ are the other treatments of the same row in the same array.

2) The second associates of $\alpha$ are the other treatments of the same column in the same array.

3) The third associates of $\alpha$ are the remaining treatments in the same array.

4) The fourth associates of $\alpha$ are the treatments in the same row and the same column as $\alpha$, of the other arrays.

5) The fifth associates of $\alpha$ are the treatments of the same row as $\alpha$ in the other arrays, that are different from the fourth associates of $\alpha$.

6) The sixth associates of $\alpha$ are the treatments of the same column as $\alpha$ in the other arrays, that are different from the fourth associates of $\alpha$.

7) The seventh associates of $\alpha$ are the remaining treatments in the other arrays.

Property 3. the parameters of generalized rectangular right angular association schemes (7) are:

$$
\begin{aligned}
& v=w n l, n_{1}=l-1, n_{2}=n-1, n_{3}=(n-1)(l-1), n_{4}=w-1 \text {, } \\
& n_{5}=(w-1)(l-1), \quad n_{6}=(w-1)(n-1), \quad n_{7}=(w-1)(n-1)(l-1) \\
& P_{1}=\left(\begin{array}{ccccccc}
l-2 & 0 & 0 & 0 & 0 & 0 & 0 \\
0 & 0 & n-1 & 0 & 0 & 0 & 0 \\
0 & n-1 & (l-2)(n-1) & 0 & 0 & 0 & 0 \\
0 & 0 & 0 & 0 & w-1 & 0 & 0 \\
0 & 0 & 0 & w-1 & (w-1)(l-2) & 0 & 0 \\
0 & 0 & 0 & 0 & 0 & 0 & (w-1)(n-1) \\
0 & 0 & 0 & 0 & 0 & (w-1)(n-1) & (w-1)(n-1)(l-2)
\end{array}\right) \\
& P_{2}=\left(\begin{array}{ccccccc}
0 & 0 & l-1 & 0 & 0 & 0 & 0 \\
0 & n-2 & 0 & 0 & 0 & 0 & 0 \\
l-1 & 0 & (l-1)(n-2) & 0 & 0 & 0 & 0 \\
0 & 0 & 0 & 0 & 0 & w-1 & 0 \\
0 & 0 & 0 & 0 & 0 & 0 & (w-1)(l-1) \\
0 & 0 & 0 & w-1 & 0 & (w-1)(n-2) & 0 \\
0 & 0 & 0 & 0 & (w-1)(l-1) & 0 & (w-1)(l-1)(n-2)
\end{array}\right) \\
& P_{3}=\left(\begin{array}{ccccccc}
0 & 1 & l-2 & 0 & 0 & 0 & 0 \\
1 & 0 & n-2 & 0 & 0 & 0 & 0 \\
l-2 & n-2 & (l-2)(n-2) & 0 & 0 & 0 & 0 \\
0 & 0 & 0 & 0 & 0 & 0 & w-1 \\
0 & 0 & 0 & 0 & 0 & w-1 & (w-1)(l-2) \\
0 & 0 & 0 & 0 & w-1 & 0 & (w-1)(n-2) \\
0 & 0 & 0 & w-1 & (w-1)(l-2) & (w-1)(n-2) & (w-1)(l-2)(n-2)
\end{array}\right) \\
& P_{4}=\left(\begin{array}{ccccccc}
0 & 0 & 0 & 0 & l-1 & 0 & 0 \\
0 & 0 & 0 & 0 & 0 & n-1 & 0 \\
0 & 0 & 0 & 0 & 0 & 0 & (l-1)(n-1) \\
0 & 0 & 0 & w-2 & 0 & 0 & 0 \\
l-1 & 0 & 0 & 0 & (w-2)(l-1) & 0 & 0 \\
0 & n-1 & 0 & 0 & 0 & (w-2)(n-1) & 0 \\
0 & 0 & (l-1)(n-1) & 0 & 0 & 0 & (w-2)(l-1)(n-1)
\end{array}\right)
\end{aligned}
$$




$$
\begin{aligned}
& P_{5}=\left(\begin{array}{ccccccc}
0 & 0 & 0 & 1 & l-2 & 0 & 0 \\
0 & 0 & 0 & 0 & 0 & 0 & n-1 \\
0 & 0 & 0 & 0 & 0 & n-1 & (l-2)(n-1) \\
1 & 0 & 0 & 0 & w-2 & 0 & 0 \\
l-2 & 0 & 0 & w-2 & (w-2)(l-2) & 0 & 0 \\
0 & 0 & n-1 & 0 & 0 & 0 & (w-2)(n-1) \\
0 & n-1 & (l-2)(n-1) & 0 & 0 & (w-2)(n-1) & (w-2)(l-2)(n-1)
\end{array}\right) \\
& P_{6}=\left(\begin{array}{ccccccc}
0 & 0 & 0 & 0 & 0 & 0 & l-1 \\
0 & 0 & 0 & 1 & 0 & n-2 & 0 \\
0 & 0 & 0 & 0 & l-1 & 0 & (l-1)(n-2) \\
0 & 1 & 0 & 0 & 0 & w-2 & 0 \\
0 & 0 & l-1 & 0 & 0 & 0 & (w-2)(l-1) \\
0 & n-2 & 0 & w-2 & 0 & (w-2)(n-2) & 0 \\
l-1 & 0 & (l-1)(n-2) & 0 & (w-2)(l-1) & 0 & (w-2)(l-1)(n-2)
\end{array}\right) \\
& P_{7}=\left(\begin{array}{ccccccc}
0 & 0 & 0 & 0 & 0 & 1 & l-2 \\
0 & 0 & 0 & 0 & 1 & 0 & n-2 \\
0 & 0 & 0 & 1 & l-2 & n-2 & (l-2)(n-2) \\
0 & 0 & 1 & 0 & 0 & 0 & w-2 \\
0 & 1 & l-2 & 0 & 0 & w-2 & (w-2)(l-2) \\
1 & 0 & n-2 & 0 & w-2 & 0 & (w-2)(n-2) \\
l-2 & n-2 & (l-2)(n-2) & w-2 & (w-2)(l-2) & (w-2)(n-2) & (w-2)(n-2)(l-2)
\end{array}\right)
\end{aligned}
$$

Definition 9. A PBIB design based on a generalized rectangular right angular association scheme (7) is called generalized rectangular right angular $\mathrm{GPBIB}_{7}$ design.

\section{Construction Method of GPBIB $_{m}$ Designs ( $m=4,5$ and 7 Associated Classes)}

Let $v=w n l(w \geq 2, n \geq 2, l \geq 2)$ treatments be arranged in $w$ arrays of $n$ rows and $l$ columns $A^{(1)}, \ldots, A^{(w)}$ and written as follows $\forall g=1, \cdots, w$ :

$$
\begin{aligned}
& R_{1}^{(g)} \begin{array}{lllllll}
C_{1}^{(g)} & C_{2}^{(g)} & \cdots & C_{j}^{(g)} & \ldots & C_{1}^{(g)} \\
\hline a_{11}^{(g)} & a_{12}^{(g)} & \ldots & a_{11}^{(g)} & \ldots & a_{11}^{(g)}
\end{array} \\
& \begin{array}{llllllll}
R_{2}^{(g)} & a_{21}^{(g)} & a_{22}^{(g)} & \ldots & a_{2 j}^{(g)} & \cdots & a_{2 l}^{(g)}
\end{array}
\end{aligned}
$$

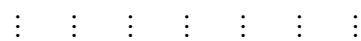

$$
\begin{aligned}
& \begin{array}{lllllll}
R_{i}^{(g)} & a_{i 1}^{(g)} & a_{i 2}^{(g)} & \ldots & a_{i j}^{(g)} & \ldots & a_{i i}^{(g)}
\end{array} \\
& \begin{array}{lllllll}
R_{n}^{(g)} & a_{n 1}^{(g)} & a_{n 2}^{(g)} & \cdots & a_{n j}^{(g)} & \cdots & a_{n 1}^{(g)} \\
\multicolumn{5}{c}{A^{(g)}} & &
\end{array}
\end{aligned}
$$

\subsection{Construction Method of $\mathrm{GPBIB}_{4}$ Designs}

\subsubsection{First Construction Method of GPBIB 4 Designs}

Applying the Combinatory Method (s) on each of the $w$ arrays, with chosen $s \in\{2, \cdots, l-1\}$, then we obtain $w$ rectangular PBIB designs. The set of all blocks gives a PBIB design with 4 associated classes.

Theorem 1. The partially balanced incomplete block designs with the parameters: 
$v=w n l, \quad b=w n(n-1) C_{s}^{l} / 2, \quad r=(n-1) C_{s-1}^{l-1}, \quad k=2 s, \quad \lambda_{1}=(n-1) C_{s-2}^{l-2}, \quad \lambda_{2}=C_{s-1}^{l-1}, \quad \lambda_{3}=C_{s-2}^{l-2}, \quad \lambda_{4}=0$

are generalized rectangular right angular $\mathrm{GPBIB}_{4}$ designs.

Proof. For each array of the $w$ arrays, we obtain a rectangular design with parameters: $v^{*}=n l$, $b^{*}=n(n-1) C_{s}^{l} / 2, \quad r=(n-1) C_{s-1}^{l-1}, \quad k=2 s, \quad \lambda_{1}=(n-1) C_{s-2}^{l-2}, \quad \lambda_{2}=C_{s-1}^{l-1}, \quad \lambda_{3}=C_{s-2}^{l-2}$. (see [5]).

For the $w$ arrays we obtain a generalized rectangular right angular GPBIB $_{4}$ design with parameters: $v=w n l$, $b=w n(n-1) C_{s}^{l} / 2, \quad r=(n-1) C_{s-1}^{l-1}, \quad k=2 s, \quad \lambda_{1}=(n-1) C_{s-2}^{l-2}, \quad \lambda_{2}=C_{s-1}^{l-1}, \quad \lambda_{3}=C_{s-2}^{l-2}$.

$\lambda_{4}$ : Two treatments $a_{i j}^{(g)}$ and $a_{i j^{\prime}}^{\left(g^{\prime}\right)}$ from the arrays $A^{(g)}$ and $A^{\left(g^{\prime}\right)}$ respectively $\left(g \neq g^{\prime} \in\{1, \cdots, w\}\right)$ they never appear together in the same block thus $\lambda_{4}=0$.

Lemma 1. For the special case $s=l$, the previous method can also be used for the construction of nested group divisible designs, with parameters:

$$
v=w n l, \quad b=w n(n-1) / 2, \quad r=(n-1), \quad k=2 l, \quad \lambda_{1}=n-1, \quad \lambda_{2}=1=\lambda_{3}, \quad \lambda_{4}=0
$$

\section{Remark 1.}

- For $w=1$, the $\mathrm{GPBIB}_{4}$ design of Theorem 1 is a rectangular design with parameters as in the Theorem 1 of [5].

- For $w=2$, the $\mathrm{GPBIB}_{4}$ design of Theorem 1 is a rectangular right angular PBIB $\mathrm{B}_{4}$ design with parameters as in Proposition 1 of [8].

Proposition 2. Let $\mathrm{GPBIB}_{4}$ be a design with parameters:

$v=w n l, \quad b=w n(n-1) C_{s}^{l} / 2, \quad r=(n-1) C_{s-1}^{l-1}, \quad k=2 s, \quad \lambda_{1}=(n-1) C_{s-2}^{l-2}, \quad \lambda_{2}=C_{s-1}^{l-1}, \quad \lambda_{3}=C_{s-2}^{l-2}, \quad \lambda_{4}=0$.

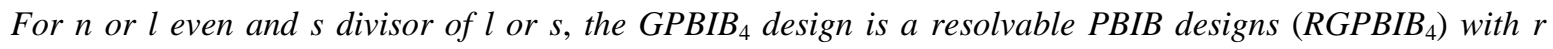
parallel classes where each parallel classes contain $q=w \frac{n l}{2 s}$ blocks.

Proof.

$$
q=b / r=\frac{w n(n-1) C_{s}^{l}}{2(n-1) C_{s-1}^{l-1}}=\frac{w n(n-1) l C_{s-1}^{l-1}}{2 s(n-1) C_{s-1}^{l-1}}=\frac{w n l}{2 s}
$$

$n$ or $l$ is even and $s$ is divisor of $n$ or $l$, then $q \in \mathbf{N}$.

Example 1. Let $v=3 \times 4 \times 4$ treatments be arranged in the three following arrays:

\begin{tabular}{cccc}
\hline 1 & 2 & 3 & 4 \\
5 & 6 & 7 & 8 \\
9 & 10 & 11 & 12 \\
13 & 14 & 15 & 16 \\
\hline & & & \\
\hline 17 & 18 & 19 & 20 \\
21 & 22 & 23 & 24 \\
25 & 26 & 27 & 28 \\
29 & 30 & 31 & 32 \\
\hline 33 & 34 & 35 & 36 \\
37 & 38 & 39 & 40 \\
41 & 42 & 43 & 44 \\
45 & 46 & 47 & 48 \\
\hline
\end{tabular}

The construction method for $(s=2)$, give the following resolvable generalized rectangular right angular $\mathrm{GPBIB}_{4}$ design, with the parameters:

$$
v=48, \quad b=108, \quad r=9, \quad k=4, \quad q=12
$$




\begin{tabular}{|c|c|c|c|}
\hline \multicolumn{4}{|c|}{$\mathrm{PC}_{1}$} \\
\hline 1 & 2 & 5 & 6 \\
\hline 9 & 10 & 13 & 14 \\
\hline 3 & 4 & 7 & 8 \\
\hline 11 & 12 & 15 & 16 \\
\hline 17 & 18 & 21 & 22 \\
\hline 25 & 26 & 29 & 30 \\
\hline 19 & 20 & 23 & 24 \\
\hline 27 & 28 & 31 & 32 \\
\hline 33 & 34 & 37 & 38 \\
\hline 35 & 36 & 39 & 40 \\
\hline 41 & 42 & 45 & 46 \\
\hline 43 & 44 & 47 & 48 \\
\hline \multicolumn{4}{|c|}{$\mathrm{PC}_{2}$} \\
\hline 1 & 2 & 9 & 10 \\
\hline 5 & 6 & 13 & 14 \\
\hline 3 & 4 & 11 & 12 \\
\hline 7 & 8 & 15 & 16 \\
\hline 17 & 18 & 25 & 26 \\
\hline 21 & 22 & 29 & 30 \\
\hline 19 & 20 & 27 & 28 \\
\hline 23 & 24 & 31 & 32 \\
\hline 33 & 34 & 41 & 42 \\
\hline 37 & 38 & 45 & 46 \\
\hline 35 & 36 & 43 & 44 \\
\hline 39 & 40 & 47 & 48 \\
\hline \multicolumn{4}{|c|}{$\mathrm{PC}_{3}$} \\
\hline 1 & 2 & 13 & 14 \\
\hline 5 & 6 & 9 & 10 \\
\hline 3 & 4 & 15 & 16 \\
\hline 7 & 8 & 11 & 12 \\
\hline 17 & 18 & 29 & 30 \\
\hline 21 & 22 & 25 & 26 \\
\hline 19 & 20 & 31 & 32 \\
\hline 23 & 24 & 27 & 28 \\
\hline 33 & 34 & 45 & 46 \\
\hline 37 & 38 & 41 & 42 \\
\hline 35 & 36 & 47 & 48 \\
\hline 39 & 40 & 43 & 44 \\
\hline
\end{tabular}




\begin{tabular}{|c|c|c|c|}
\hline \multicolumn{4}{|c|}{$\mathrm{PC}_{4}$} \\
\hline 1 & 3 & 5 & 7 \\
\hline 9 & 11 & 13 & 15 \\
\hline 2 & 4 & 6 & 8 \\
\hline 10 & 12 & 14 & 16 \\
\hline 17 & 19 & 21 & 23 \\
\hline 25 & 27 & 29 & 31 \\
\hline 18 & 20 & 22 & 24 \\
\hline 26 & 28 & 30 & 32 \\
\hline 33 & 35 & 37 & 39 \\
\hline 34 & 37 & 38 & 40 \\
\hline 41 & 43 & 45 & 47 \\
\hline 42 & 44 & 46 & 48 \\
\hline \multicolumn{4}{|c|}{$\mathrm{PC}_{5}$} \\
\hline 1 & 3 & 9 & 11 \\
\hline 5 & 7 & 13 & 15 \\
\hline 2 & 4 & 10 & 12 \\
\hline 6 & 8 & 14 & 16 \\
\hline 17 & 19 & 25 & 27 \\
\hline 21 & 23 & 29 & 31 \\
\hline 18 & 20 & 26 & 28 \\
\hline 22 & 24 & 30 & 32 \\
\hline 33 & 35 & 41 & 43 \\
\hline 37 & 39 & 45 & 47 \\
\hline 34 & 36 & 42 & 44 \\
\hline 38 & 40 & 46 & 48 \\
\hline \multicolumn{4}{|c|}{$\mathrm{PC}_{6}$} \\
\hline 1 & 3 & 13 & 15 \\
\hline 5 & 7 & 9 & 11 \\
\hline 2 & 4 & 14 & 16 \\
\hline 6 & 8 & 10 & 12 \\
\hline 17 & 19 & 29 & 31 \\
\hline 21 & 23 & 25 & 27 \\
\hline 18 & 20 & 30 & 32 \\
\hline 22 & 24 & 26 & 28 \\
\hline 33 & 35 & 45 & 47 \\
\hline 37 & 39 & 41 & 43 \\
\hline 34 & 36 & 46 & 48 \\
\hline 38 & 40 & 42 & 44 \\
\hline
\end{tabular}




\begin{tabular}{|c|c|c|c|}
\hline \multicolumn{4}{|c|}{$\mathrm{PC}_{7}$} \\
\hline 1 & 4 & 5 & 8 \\
\hline 9 & 12 & 13 & 16 \\
\hline 2 & 3 & 6 & 7 \\
\hline 10 & 11 & 14 & 15 \\
\hline 17 & 20 & 21 & 24 \\
\hline 25 & 28 & 29 & 32 \\
\hline 18 & 19 & 22 & 23 \\
\hline 26 & 27 & 30 & 31 \\
\hline 33 & 36 & 37 & 40 \\
\hline 34 & 35 & 38 & 39 \\
\hline 41 & 44 & 45 & 48 \\
\hline 42 & 43 & 46 & 47 \\
\hline \multicolumn{4}{|c|}{$\mathrm{PC}_{8}$} \\
\hline 1 & 4 & 9 & 12 \\
\hline 5 & 8 & 13 & 16 \\
\hline 2 & 3 & 10 & 11 \\
\hline 6 & 7 & 14 & 15 \\
\hline 17 & 20 & 25 & 28 \\
\hline 21 & 24 & 29 & 32 \\
\hline 18 & 19 & 26 & 27 \\
\hline 22 & 23 & 30 & 31 \\
\hline 33 & 36 & 41 & 44 \\
\hline 37 & 40 & 45 & 48 \\
\hline 34 & 35 & 42 & 43 \\
\hline 38 & 39 & 46 & 47 \\
\hline \multicolumn{4}{|c|}{$\mathrm{PC}_{9}$} \\
\hline 1 & 4 & 13 & 16 \\
\hline 5 & 8 & 9 & 12 \\
\hline 2 & 3 & 14 & 15 \\
\hline 6 & 7 & 10 & 11 \\
\hline 17 & 20 & 29 & 32 \\
\hline 21 & 24 & 25 & 28 \\
\hline 18 & 19 & 30 & 31 \\
\hline 22 & 23 & 26 & 27 \\
\hline 33 & 36 & 45 & 48 \\
\hline 37 & 40 & 41 & 44 \\
\hline 34 & 35 & 46 & 47 \\
\hline 38 & 39 & 42 & 43 \\
\hline
\end{tabular}




\subsubsection{Second Construction Method of GPBIB 4 Designs with $\lambda_{4} \neq 0$}

Let $C_{j}^{(g)}=\left(a_{1 j}^{(g)}, a_{2 j}^{(g)}, \cdots, a_{n j}^{(g)}\right)^{\prime}, \quad C_{j}^{(g) 1}=C_{j}^{(g)}$ and $C_{j}^{(g) h}=\left(a_{h j}^{(g)}, a_{(h+1) j}^{(g)}, \cdots, a_{n j}^{(g)}, a_{1 j}^{(g)}, \cdots, a_{(h-1) j}^{(g)}\right)^{\prime}, \quad h=2,3, \cdots, n$. Applying the Combinatory Method $(s)$ with chosen $s \in\{3, \cdots, l\}$ on each array of the form $C_{j}^{(g) h} \cup A^{\left(g^{\prime}\right)}$ $\left(g^{\prime} \neq g\right)$ for $j=1, \cdots, l, h=1, \cdots, n$ and $g=1, \cdots, w$, by only considering the combinations of $s$ treatments that always contain a component of the vector $C_{j}^{(g) h}$, the set of all the obtained blocks provides a PBIP with 4 associated classes.

Theorem 3. The partially balanced incomplete block designs with the parameters:

$$
\begin{gathered}
v=w n l, \quad b=w(w-1) \ln ^{2}(n-1) C_{s-1}^{l} / 2, \quad r=s(w-1) n(n-1) C_{s-1}^{l}, \quad k=2 s, \quad \lambda_{1}=(w-1) \ln (n-1) C_{s-3}^{l-2}, \\
\lambda_{2}=s(w-1) n C_{s-1}^{l}, \quad \lambda_{3}=(w-1) n l C_{s-3}^{l-2}, \quad \lambda_{4}=4(n-1) C_{s-2}^{l-1}
\end{gathered}
$$

are generalized rectangular right angular $\mathrm{GPBIB}_{4}$ designs.

Proof.

- The $v$ and $k$ values are obvious.

- $r$ : For each treatment $a_{i j_{0}}^{(g)}$ of the array $A^{(g)} g=1, \cdots, w$, we have:

$\circ$ On an array $C_{j_{0}}^{(g) h} \cup A^{\left(g^{\prime}\right)}\left(g^{\prime} \neq g\right)$, applying the procedure with the $l$ other elements of the same row. There is $C_{s-1}^{l}$ possibilities, each one being repeated $(n-1)$ times, with $n$ permutations $(h=1, \cdots, n)$, then we have $n(n-1) C_{s-1}^{l}$ repetitions. Therefore, we have $w-1$ arrays of the form $C_{j_{0}}^{(g) h} \cup A^{\left(g^{\prime}\right)}$ $\left(g^{\prime} \neq g\right)$; so we have $(w-1) n(n-1) C_{s-1}^{l}$ repetitions of the treatment $a_{i j_{0}}^{(g)}$.

$\circ$ On an array $A^{(g)} \cup C_{j}^{\left(g^{\prime}\right) h}\left(g^{\prime} \neq g\right), \quad j=1, \cdots, l$ and $h=1, \cdots, n$, applying the procedure with the $l-1$ other elements of the same row. There is $C_{s-2}^{l-1}$ possibilities each one being repeated $(n-1)$ times, with $n$ permutations $(h=1, \cdots, n)$, then we have $(n-1) C_{s-2}^{l-1}$ appearances repeated themselves $n$ times. Therefore, for an array $A^{(g)} \cup C_{j}^{\left(g^{\prime}\right) h}$ we have $n(n-1) C_{s-2}^{l-1}$ repetitions of $a_{i j_{0}}$. Considering all the arrays $A^{(g)} \cup C_{j}^{\left(g^{\prime}\right) h}\left(g^{\prime} \neq g\right)$ for $j=1, \cdots, l$ and $g^{\prime}=1, \cdots, w$; so we have $(w-1) \ln (n-1) C_{s-2}^{l-1}$ repetitions of $a_{i j_{0}}$.

Thus: $r=(w-1) n(n-1)\left[C_{s-1}^{l}+l C_{s-2}^{l-1}\right]=s(w-1) n(n-1) C_{s-1}^{l}$.

- $\lambda_{1}$ : Consider two treatments $a_{i j_{0}}^{(g)}$ and $a_{i j_{0}^{\prime}}^{(g)}$ from the array $A^{(g)}\left(g=1, \cdots, w\right.$ and $\left.j_{0} \neq j_{0}^{\prime}\right)$, they appear together $(n-1) C_{s-3}^{l-2}$ times with the other $l-2$ elements of the same row $i$ of the arrays $A^{(g)} \cup C_{j}^{\left(g^{\prime}\right) h}$ $\left(g^{\prime} \neq g\right)$, with $n$ permutations $(h=1, \cdots, n)$, we obtain $n(n-1) C_{s-3}^{l-2}$ times in which the two treatments appear together. Considering all the arrays $A^{(g)} \cup C_{j}^{\left(g^{\prime}\right) h}\left(g^{\prime} \neq g\right)$ for $j=1, \cdots, l$ and $g^{\prime}=1, \cdots, w$; then we have $(w-1) \ln (n-1) C_{s-3}^{l-2}$ times where the two treatments $a_{i j_{0}}$ and $a_{i j^{\prime}}$ appear together.

- $\quad \lambda_{2}$ : Consider two treatments $a_{i j_{0}}^{(g)}$ and $a_{i^{\prime} j_{0}}^{(g)}$ from the array $A^{(g)},\left(g=1, \cdots, w\right.$ and $\left.i \neq i^{\prime}\right)$, we have:

$\circ$ In an array $A^{(g)} \cup C_{j}^{\left(g^{\prime}\right) h}\left(g^{\prime} \neq g\right)$ : the two treatments appear together $C_{s-2}^{l-1}$ times, with the $n$ permutations $(h=1, \cdots, n)$ they appear together $n C_{s-2}^{l-1}$ times. Considering all the arrays of the form $A^{(g)} \cup C_{j}^{\left(g^{\prime}\right) h}\left(g^{\prime} \neq g\right)$ for $j=1, \cdots, l$ and $g^{\prime}=1, \cdots, w$, they appear together $(w-1) \ln C_{s-2}^{l-1}$ times.

○ In the array $C_{j_{0}}^{(g) h} \cup A^{\left(g^{\prime}\right)}$ : both treatments appear together $C_{s-1}^{l}$ times, with the $n$ permutations $(h=1, \cdots, n)$ they appear $n C_{s-1}^{l}$ times. Considering all the arrays of the form $C_{j_{0}}^{(g) h} \cup A^{\left(g^{\prime}\right)} \quad\left(g^{\prime} \neq g\right)$ for $g^{\prime}=1, \cdots, w$; they appear together $(w-1) n C_{s-1}^{l}$ times.

In total $\lambda_{2}=(w-1) n\left[l C_{s-2}^{l-1}+C_{s-1}^{l}\right]=s n(w-1) C_{s-1}^{l}$.

- $\lambda_{3}$ : Consider two treatments $a_{i j_{0}}^{(g)}$ and $a_{i^{\prime} j_{0}^{\prime}}^{(g)}$ from the array $A^{(g)}\left(g=1, \cdots, w, i \neq i^{\prime}\right.$ and $\left.j_{0} \neq j_{0}^{\prime}\right)$, they appear together $C_{s-3}^{l-2}$ times for each array of the form $A^{(g)} \cup C_{j}^{\left(g^{\prime}\right) h}\left(g^{\prime} \neq g\right)$, with the $n$ permutations $(h=1, \cdots, n)$ they appear together $n C_{s-3}^{l-2}$ times. Considering all the arrays $A^{(g)} \cup C_{j}^{\left(g^{\prime}\right) h}\left(g^{\prime} \neq g\right)$ for $j=1, \cdots, l$ and $g^{\prime}=1, \cdots, w$, then the two treatments $a_{i j_{0}}^{(g)}$ and $a_{i j_{0}^{\prime}}^{(g)}$ appear together $(w-1) \ln C_{s-3}^{l-2}$ times.

- $\lambda_{4}$ : Consider two treatments $a_{i j}^{(g)}$ and $a_{i j^{\prime}}^{\left(g^{\prime}\right)}$ from the arrays $A^{(g)}$ and $A^{\left(g^{\prime}\right)}$ respectively $\left(g, g^{\prime}=1, \cdots, w\right)$ :

$\circ$ If $i=i^{\prime}$, for the array $A^{(g)^{\prime j}} \cup C_{j}^{\left(g^{\prime}\right) h}\left(g^{\prime} \neq g\right)$ and $h=1$ the two treatments appear together $(n-1) C_{s-2}^{l-1}$ times. For the array $C_{j}^{(g) h} \cup A^{\left(g^{\prime}\right)}\left(g^{\prime} \neq g\right)$ and $h=1$ they also appear together $(n-1) C_{s-2}^{l-1}$ time, so we have $2(n-1) C_{s-2}^{l-1}$ times. On the other hand, for $h=2, \cdots, n$, the two treatments appear together $C_{s-2}^{l-1}$ times for the array $A^{(g)} \cup C_{j}^{\left(g^{\prime}\right) h}$ and $C_{s-2}^{l-1}$ times for the array $C_{j}^{(g) h} \cup A^{\left(g^{\prime}\right)}$, so we have $2 C_{s-2}^{l-1}$ for one value of $h$. Taking all the values of $h=2, \cdots, n$, we obtain $2(n-1) C_{s-2}^{l-1}$ times where the two treatments appear together. 
In total $\lambda_{4}=2(n-1) C_{s-2}^{l-1}+2(n-1) C_{s-2}^{l-1}=4(n-1) C_{s-2}^{l-1}$.

○ If $i \neq i^{\prime}$, then the two treatments appear together $C_{s-2}^{l-1}$ times for the array of the form $A^{(g)} \cup C_{j^{\prime}}^{(g) h}$ $\left(g^{\prime} \neq g\right)$ and appear together $C_{s-2}^{l-1}$ times for the array of the form $C_{j}^{(g) h} \cup A^{\left(g^{\prime}\right)} \quad\left(g^{\prime} \neq g\right)$ for $h=1$, so we have $2 C_{s-2}^{l-1}$ times. For $h=2, \cdots, n$, among the $(n-1)$ permutations of the vector $C_{j^{\prime}}^{(g) h}$, and for a given value of $h$, the treatment $a_{i j^{\prime}}^{\left(g^{\prime}\right)}$ takes the same row as $a_{i j}^{(g)}$ then the two treatments appear together $(n-1) C_{s-2}^{l-1}$ times, for the remaining values the two treatments appear together $C_{s-2}^{l-1}$ times. For $h=2, \cdots, n$, among the $(n-1)$ permutations of the vector $C_{j^{\prime}}^{(g)}$ and for a given value of $h$, the treatment $a_{i j}^{(g)}$ takes the same row as $a_{i^{\prime} j^{\prime}}^{\left(g^{\prime}\right)}$ then the two treatments appear together $(n-1) C_{s-2}^{l-1}$ times, for the remaining values the two treatments appear together $C_{s-2}^{l-1}$ times.

In total $\lambda_{4}=2 C_{s-2}^{l-1}+(n-1) C_{s-2}^{l-1}+(n-1) C_{s-2}^{l-1}+(n-1) C_{s-2}^{l-1}+(n-1) C_{s-2}^{l-1}=4(n-1) C_{s-2}^{l-1}$.

- $b$ : Using the above construction method on each array of the form: $A^{(g)} \cup C_{j^{\prime}}^{(g) h}\left(g^{\prime} \neq g\right)$, we obtain $n(n-1) C_{s-1}^{l} / 2$ blocks. So for the $l$ arrays of the form $A \cup C_{j}^{(g) h}$ we have $\ln (n-1) C_{s-1}^{l} / 2$ blocks, with the $n$ permutations $(h=1, \cdots, n)$ we obtain $\ln ^{2}(n-1) C_{s-1}^{l} / 2$ blocks. Considering all the arrays $A^{(g)} \cup C_{j}^{\left(g^{\prime}\right) h}$ for $g^{\prime}=1, \cdots, w$, then in total we have $(w-1) \ln ^{2}(n-1) C_{s-1}^{l} / 2$ blocks. Considering all the arrays $A^{(g)} \cup C_{j}^{\left(g^{\prime}\right) h}$ for $g=1, \cdots, w$, then in total we have $w(w-1) \ln ^{2}(n-1) C_{s-1}^{l} / 2$ blocks.

Remark 2. For $w=2$, the $\mathrm{GPBIB}_{4}$ design of Theorem 3 is a rectangular right angular $\mathrm{PBIB}_{4}$ design with parameters as in Proposition 2 of [8].

Proposition 4 Let $\mathrm{GPBIB}_{4}$ be a design with parameters:

$$
\begin{gathered}
v=w n l, \quad b=w(w-1) \ln ^{2}(n-1) C_{s-1}^{l} / 2, \quad r=s(w-1) n(n-1) C_{s-1}^{l}, \quad k=2 s, \quad \lambda_{1}=(w-1) \ln (n-1) C_{s-3}^{l-2}, \\
\lambda_{2}=s(w-1) n C_{s-1}^{l}, \quad \lambda_{3}=(w-1) n l C_{s-3}^{l-2}, \quad \lambda_{4}=4(n-1) C_{s-2}^{l-1}
\end{gathered}
$$

For $n$ or $l$ even and s divisor of $l$ or $s$, the $\mathrm{GPBIB}_{4}$ design is a resolvable PBIB designs $\left(R G P B I B_{4}\right)$ with $r$ parallel classes where each parallel classes contain $q=w \frac{n l}{2 s}$ blocks.

\subsection{Construction Method of GPBIB 5 Designs}

Let $C_{j}^{(g)}=\left(a_{1 j}^{(g)}, a_{2 j}^{(g)}, \cdots, a_{n j}^{(g)}\right)^{\prime}$ be the $j^{\text {th }}$ column of the $g^{\text {th }}$ array $g \in\{1, \cdots, w\}$. Applying the Combinatory Method (s) with $s$ chosen $s \in\{3, \cdots, l\}$, on each array in the form $C_{j}^{(g)} \cup A^{\left(g^{\prime}\right)} \quad\left(g \neq g^{\prime}\right)$ for $j=1, \cdots, l$ and $j=1, \cdots, w$ by only considering the combinations of $s$ treatments that contain a component of the vector $C_{j}^{(g)}$, then the set of all the blocks obtained, gives a PBIB design with 5 associated classes.

Theorem 5. The incomplete block designs with parameters:

$$
\begin{gathered}
v=w n l, \quad b=w(w-1) \ln (n-1) C_{s-1}^{l} / 2, \quad r=s(w-1)(n-1) C_{s-1}^{l}, \quad k=2 s, \quad \lambda_{1}=(w-1) l(n-1) C_{s-3}^{l-2}, \\
\lambda_{2}=(w-1) s C_{s-1}^{l}, \quad \lambda_{3}=(w-1) l C_{s-3}^{l-2}, \quad \lambda_{4}=2(n-1) C_{s-2}^{l-1}, \quad \lambda_{5}=2 C_{s-2}^{l-1}
\end{gathered}
$$

are generalized rectangular right angular $\mathrm{GPBIB}_{5}$ designs.

Remark 3. For $w=2$, the $G P B I B_{5}$ design of Theorem 5 is a rectangular right angular $\mathrm{PBIB}_{5}$ design with parameters as in Proposition 3 of [8].

Proposition 6. Let $G P B I B_{5}$ be a design with parameters:

$$
\begin{gathered}
v=w n l, \quad b=w(w-1) \ln (n-1) C_{s-1}^{l} / 2, \quad r=s(w-1)(n-1) C_{s-1}^{l}, \quad k=2 s, \quad \lambda_{1}=(w-1) l(n-1) C_{s-3}^{l-2}, \\
\lambda_{2}=(w-1) s C_{s-1}^{l}, \quad \lambda_{3}=(w-1) l C_{s-3}^{l-2}, \quad \lambda_{4}=2(n-1) C_{s-2}^{l-1}, \quad \lambda_{5}=2 C_{s-2}^{l-1}
\end{gathered}
$$

For $n$ or $l$ even and s divisor of $l$ or $s$, the GPBIB $B_{5}$ design is a resolvable PBIB designs (RGPBIB $\left.B_{5}\right)$ with $r$ parallel classes where each parallel classes contain $q=w \frac{n l}{2 s}$ blocks.

Example 2. Let $v=3 \times 2 \times 3$ treatments be arranged in the two following arrays: 


\begin{tabular}{ccc}
\hline 1 & 2 & 3 \\
4 & 5 & 6 \\
\hline 7 & 8 & 9 \\
10 & 11 & 12 \\
\hline 13 & 14 & 15 \\
16 & 17 & 18 \\
\hline
\end{tabular}

The construction method for $(s=3)$, give the following generalized rectangular right angular $G P B I B_{5}$ design, with parameters:

\begin{tabular}{|c|c|c|c|c|c|}
\hline \multicolumn{6}{|c|}{$\mathrm{PC}_{1}$} \\
\hline 1 & 7 & 8 & 4 & 10 & 11 \\
\hline 9 & 13 & 14 & 12 & 16 & 17 \\
\hline 15 & 2 & 3 & 18 & 5 & 6 \\
\hline \multicolumn{6}{|c|}{$\mathrm{PC}_{2}$} \\
\hline 1 & 7 & 9 & 4 & 10 & 12 \\
\hline 8 & 13 & 15 & 11 & 16 & 18 \\
\hline 14 & 2 & 3 & 17 & 5 & 6 \\
\hline \multicolumn{6}{|c|}{$\mathrm{PC}_{3}$} \\
\hline 1 & 8 & 9 & 4 & 11 & 12 \\
\hline 7 & 14 & 15 & 10 & 17 & 18 \\
\hline 13 & 2 & 3 & 16 & 5 & 6 \\
\hline \multicolumn{6}{|c|}{$\mathrm{PC}_{4}$} \\
\hline 2 & 7 & 8 & 5 & 10 & 11 \\
\hline 9 & 13 & 15 & 12 & 16 & 18 \\
\hline 14 & 1 & 3 & 17 & 4 & 6 \\
\hline \multicolumn{6}{|c|}{$\mathrm{PC}_{5}$} \\
\hline 2 & 7 & 9 & 5 & 10 & 12 \\
\hline 13 & 1 & 3 & 16 & 4 & 6 \\
\hline 8 & 14 & 15 & 11 & 17 & 18 \\
\hline \multicolumn{6}{|c|}{$\mathrm{PC}_{6}$} \\
\hline 2 & 8 & 9 & 5 & 11 & 12 \\
\hline 7 & 13 & 14 & 10 & 16 & 17 \\
\hline 15 & 1 & 3 & 18 & 4 & 6 \\
\hline
\end{tabular}




\begin{tabular}{|c|c|c|c|c|c|}
\hline \multicolumn{6}{|c|}{$\mathrm{PC}_{7}$} \\
\hline 3 & 7 & 8 & 6 & 10 & 11 \\
\hline 9 & 14 & 15 & 12 & 17 & 18 \\
\hline 13 & 1 & 2 & 16 & 4 & 5 \\
\hline \multicolumn{6}{|c|}{$\mathrm{PC}_{8}$} \\
\hline 3 & 7 & 9 & 6 & 10 & 12 \\
\hline 8 & 13 & 14 & 11 & 16 & 17 \\
\hline 15 & 1 & 2 & 18 & 4 & 5 \\
\hline \multicolumn{6}{|c|}{$\mathrm{PC}_{9}$} \\
\hline 3 & 8 & 9 & 6 & 11 & 12 \\
\hline 7 & 13 & 15 & 10 & 16 & 18 \\
\hline 14 & 1 & 2 & 17 & 4 & 5 \\
\hline \multicolumn{6}{|c|}{$\mathrm{PC}_{10}$} \\
\hline 13 & 7 & 8 & 16 & 10 & 11 \\
\hline 9 & 1 & 2 & 12 & 4 & 5 \\
\hline 3 & 14 & 15 & 6 & 17 & 18 \\
\hline \multicolumn{6}{|c|}{$\mathrm{PC}_{11}$} \\
\hline 13 & 7 & 9 & 16 & 10 & 12 \\
\hline 8 & 1 & 3 & 11 & 4 & 6 \\
\hline 2 & 14 & 15 & 5 & 17 & 18 \\
\hline \multicolumn{6}{|c|}{$\mathrm{PC}_{12}$} \\
\hline 13 & 8 & 9 & 16 & 11 & 12 \\
\hline 7 & 2 & 3 & 10 & 5 & 6 \\
\hline 1 & 14 & 15 & 4 & 17 & 18 \\
\hline \multicolumn{6}{|c|}{$\mathrm{PC}_{13}$} \\
\hline 14 & 7 & 8 & 17 & 10 & 11 \\
\hline 9 & 2 & 3 & 12 & 5 & 6 \\
\hline 1 & 13 & 15 & 4 & 16 & 18 \\
\hline \multicolumn{6}{|c|}{$\mathrm{PC}_{14}$} \\
\hline 14 & 7 & 9 & 17 & 10 & 12 \\
\hline 8 & 1 & 2 & 11 & 4 & 5 \\
\hline 3 & 13 & 15 & 6 & 16 & 18 \\
\hline
\end{tabular}




\begin{tabular}{|c|c|c|c|c|c|}
\hline \multicolumn{6}{|c|}{$\mathrm{PC}_{15}$} \\
\hline 14 & 8 & 9 & 17 & 11 & 12 \\
\hline 7 & 1 & 3 & 10 & 4 & 6 \\
\hline 2 & 13 & 15 & 5 & 16 & 18 \\
\hline \multicolumn{6}{|c|}{$\mathrm{PC}_{16}$} \\
\hline 15 & 7 & 8 & 18 & 10 & 11 \\
\hline 9 & 1 & 3 & 12 & 4 & 6 \\
\hline 2 & 13 & 14 & 5 & 16 & 17 \\
\hline \multicolumn{6}{|c|}{$\mathrm{PC}_{17}$} \\
\hline 15 & 7 & 9 & 18 & 10 & 12 \\
\hline 8 & 2 & 3 & 11 & 5 & 6 \\
\hline 1 & 13 & 14 & 4 & 16 & 17 \\
\hline \multicolumn{6}{|c|}{$\mathrm{PC}_{18}$} \\
\hline 15 & 8 & 9 & 18 & 11 & 12 \\
\hline 7 & 1 & 2 & 10 & 4 & 5 \\
\hline 3 & 13 & 14 & 6 & 16 & 17 \\
\hline
\end{tabular}

\subsection{Construction Method of $\mathrm{GPBIB}_{7}$ Designs}

\subsubsection{First Construction Method of GPBIB 7 Designs}

Applying the Combinatory Method (s) on each of the $w$ arrays, with chosen and fixed $s \in\{2, \cdots, l-1\}$, then we obtain $w$ rectangular PBIB designs. The juxtaposition of the blocks of the $w$ rectangular PBIB designs, such that the blocks containing treatment $a_{i j}^{(g)}$ and $a_{i j}^{\left(g^{\prime}\right)} \quad\left(g \neq g^{\prime} \in\{1, \cdots, w\}\right)$ are put side by side, gives a PBIB design with 7 associated classes.

Theorem 7. The incomplete block designs with parameters:

$$
\begin{gathered}
v=w n l, \quad b=\frac{n(n-1) C_{s}^{l}}{2}, \quad \lambda_{0}=r=(n-1) C_{s-1}^{l-1}=\lambda_{4}, \quad k=2 s w, \\
\lambda_{1}=(n-1) C_{s-2}^{l-2}=\lambda_{5}, \quad \lambda_{2}=C_{s-1}^{l-1}=\lambda_{6}, \quad \lambda_{3}=C_{s-2}^{l-2}=\lambda_{7}
\end{gathered}
$$

are generalized rectangular right angular $\mathrm{GPBIB}_{7}$ designs.

Proof. The design parameters are deduced from the construction method.

Remark 4.

- $F o r w=1$, the $G P B I B_{7}$ design of Theorem 7 is a rectangular design with parameters as in Theorem 1 of [5].

- For $w=2$, the $G P B I B_{7}$ design of Theorem 7 is a rectangular right angular PBIB ${ }_{7}$ design with parameters as in Proposition 4 of [8].

Proposition 8. Let $\mathrm{GPBIB}_{7}$ be a design with parameters:

$$
\begin{gathered}
v=w n l, \quad b=\frac{n(n-1) C_{s}^{l}}{2}, \quad \lambda_{0}=r=(n-1) C_{s-1}^{l-1}=\lambda_{4}, \quad k=2 s w, \\
\lambda_{1}=(n-1) C_{s-2}^{l-2}=\lambda_{5}, \quad \lambda_{2}=C_{s-1}^{l-1}=\lambda_{6}, \quad \lambda_{3}=C_{s-2}^{l-2}=\lambda_{7}
\end{gathered}
$$

For $n$ or $l$ even and s divisor of $l$ or $s$, the $\mathrm{GPBIB}_{7}$ design is a resolvable PBIB designs $\left(R G P B I B_{7}\right)$ with $r$ 
parallel classes where each parallel classes contain $q=\frac{n l}{2 s}$ blocks.

Example 3. Let $v=3 \times 4 \times 4$ treatments be arranged in the three following arrays:

\begin{tabular}{cccc}
\hline 1 & 2 & 3 & 4 \\
5 & 6 & 7 & 8 \\
9 & 10 & 11 & 12 \\
13 & 14 & 15 & 16 \\
\hline 17 & 18 & 19 & 20 \\
21 & 22 & 23 & 24 \\
25 & 26 & 27 & 28 \\
29 & 30 & 31 & 32 \\
\hline 33 & 34 & 35 & 36 \\
37 & 38 & 39 & 40 \\
41 & 42 & 43 & 44 \\
45 & 46 & 47 & 48 \\
\hline
\end{tabular}

The construction method for $(s=2)$, give the following resolvable generalized rectangular right angular $\mathrm{GPBIB}_{7}$ design, with the parameters:

$$
v=48, \quad b=36, \quad r=9, \quad k=12, \quad q=4
$$

\begin{tabular}{ccccccccccccc}
\hline \multicolumn{10}{c}{$\mathrm{PC}_{1}$} \\
\hline 1 & 2 & 5 & 6 & 17 & 18 & 21 & 22 & 33 & 34 & 37 & 38 \\
9 & 10 & 13 & 14 & 25 & 26 & 29 & 30 & 41 & 42 & 45 & 46 \\
3 & 4 & 7 & 8 & 19 & 20 & 23 & 24 & 35 & 36 & 39 & 40 \\
11 & 12 & 15 & 16 & 27 & 28 & 31 & 32 & 43 & 44 & 47 & 48 \\
\hline
\end{tabular}

\begin{tabular}{|c|c|c|c|c|c|c|c|c|c|c|c|}
\hline \multicolumn{12}{|c|}{$\mathrm{PC}_{2}$} \\
\hline 1 & 2 & 9 & 10 & 17 & 18 & 25 & 26 & 33 & 34 & 41 & 42 \\
\hline 5 & 6 & 13 & 14 & 21 & 22 & 29 & 30 & 37 & 38 & 45 & 46 \\
\hline 3 & 4 & 11 & 12 & 19 & 20 & 27 & 28 & 35 & 36 & 43 & 44 \\
\hline 7 & 8 & 15 & 16 & 23 & 24 & 31 & 32 & 39 & 40 & 47 & 48 \\
\hline \multicolumn{12}{|c|}{$\mathrm{PC}_{3}$} \\
\hline 1 & 2 & 13 & 14 & 17 & 18 & 29 & 30 & 33 & 34 & 45 & 46 \\
\hline 5 & 6 & 9 & 10 & 21 & 22 & 25 & 26 & 37 & 38 & 41 & 42 \\
\hline 3 & 4 & 15 & 16 & 19 & 20 & 31 & 32 & 35 & 36 & 47 & 48 \\
\hline 7 & 8 & 11 & 12 & 23 & 24 & 27 & 28 & 39 & 40 & 43 & 44 \\
\hline
\end{tabular}




\begin{tabular}{ccccccccccccc}
\hline \multicolumn{10}{c}{$\mathrm{PC}_{4}$} \\
1 & 3 & 5 & 7 & 17 & 19 & 21 & 23 & 33 & 35 & 37 & 39 \\
9 & 11 & 13 & 15 & 25 & 27 & 29 & 31 & 34 & 37 & 38 & 40 \\
2 & 4 & 6 & 8 & 18 & 20 & 22 & 24 & 41 & 43 & 45 & 47 \\
10 & 12 & 14 & 16 & 26 & 28 & 30 & 32 & 42 & 44 & 46 & 48 \\
\hline
\end{tabular}

\begin{tabular}{llllllllllll}
\hline \multicolumn{10}{c}{$\mathrm{PC}_{5}$} \\
1 & 3 & 9 & 11 & 17 & 19 & 25 & 27 & 33 & 35 & 41 & 43 \\
5 & 7 & 13 & 15 & 21 & 23 & 29 & 31 & 37 & 39 & 45 & 47 \\
2 & 4 & 10 & 12 & 18 & 20 & 26 & 28 & 34 & 36 & 42 & 44 \\
6 & 8 & 14 & 16 & 22 & 24 & 30 & 32 & 38 & 40 & 46 & 48 \\
\hline
\end{tabular}

\begin{tabular}{|c|c|c|c|c|c|c|c|c|c|c|c|}
\hline \multicolumn{12}{|c|}{$\mathrm{PC}_{6}$} \\
\hline 1 & 3 & 13 & 15 & 17 & 19 & 29 & 31 & 33 & 35 & 45 & 47 \\
\hline 5 & 7 & 9 & 11 & 21 & 23 & 25 & 27 & 37 & 39 & 41 & 43 \\
\hline 2 & 4 & 14 & 16 & 18 & 20 & 30 & 32 & 34 & 36 & 46 & 48 \\
\hline 6 & 8 & 10 & 12 & 22 & 24 & 26 & 28 & 38 & 40 & 42 & 44 \\
\hline \multicolumn{12}{|c|}{$\mathrm{PC}_{7}$} \\
\hline 1 & 4 & 5 & 8 & 17 & 20 & 21 & 24 & 33 & 36 & 37 & 40 \\
\hline 9 & 12 & 13 & 16 & 25 & 28 & 29 & 32 & 34 & 35 & 38 & 39 \\
\hline 2 & 3 & 6 & 7 & 18 & 19 & 22 & 23 & 41 & 44 & 45 & 48 \\
\hline 10 & 11 & 14 & 15 & 26 & 27 & 30 & 31 & 42 & 43 & 46 & 47 \\
\hline
\end{tabular}

\begin{tabular}{llllllllllll}
\hline \multicolumn{10}{c}{$\mathrm{PC}_{8}$} \\
1 & 4 & 9 & 12 & 17 & 20 & 25 & 28 & 33 & 36 & 41 & 44 \\
5 & 8 & 13 & 16 & 21 & 24 & 29 & 32 & 37 & 40 & 45 & 48 \\
2 & 3 & 10 & 11 & 18 & 19 & 26 & 27 & 34 & 35 & 42 & 43 \\
6 & 7 & 14 & 15 & 22 & 23 & 30 & 31 & 38 & 39 & 46 & 47 \\
\hline
\end{tabular}

\begin{tabular}{|c|c|c|c|c|c|c|c|c|c|c|c|}
\hline \multicolumn{12}{|c|}{$\mathrm{PC}_{9}$} \\
\hline 1 & 4 & 13 & 16 & 17 & 20 & 29 & 32 & 33 & 36 & 45 & 48 \\
\hline 5 & 8 & 9 & 12 & 21 & 24 & 25 & 28 & 37 & 40 & 41 & 44 \\
\hline 2 & 3 & 14 & 15 & 18 & 19 & 30 & 31 & 34 & 35 & 46 & 47 \\
\hline 6 & 7 & 10 & 11 & 22 & 23 & 26 & 27 & 38 & 39 & 42 & 43 \\
\hline
\end{tabular}

3.3.2. Second Construction Method of GPBIB 7 Designs with $\lambda_{i} \neq \lambda_{i+4} \quad(i=0, \cdots, 4)$

Let $C_{j}^{(g)}=\left(a_{1 j}^{(g)}, a_{2 j}^{(g)}, \cdots, a_{n j}^{(g)}\right)^{\prime}$ be the $j^{\text {th }}$ column of the $g^{\text {th }}$ array and let $R_{i}^{(g)}=\left(a_{i 1}^{(g)}, a_{i 2}^{(g)}, \cdots, a_{i l}^{(g)}\right)$ be the $i^{\text {th }}$ row of the $g^{\text {th }}$ array $(g \in\{1, \cdots, w\})$. Then applying the Combinatory Method (s) with chosen $s \in\{2, \cdots, l\}$ on each 
array of the form $C_{j}^{(g)} \cup\left[A^{\left(g^{\prime}\right)} \backslash C_{j}^{\left(g^{\prime}\right)}\right] \quad\left(g^{\prime} \neq g\right)$ for $j=1, \cdots, l$ and $g, g^{\prime}=1, \cdots, w$ (respectively $R_{i}^{(g)} \cup\left[A^{\left(g^{\prime}\right)} \backslash R_{i}^{\left(g^{\prime}\right)}\right] \quad\left(g^{\prime} \neq g\right)$ for $i=1, \cdots, n$ and $\left.g, g^{\prime}=1, \cdots, w\right)$, by only considering the combinations of $s$ treatments that always contain a component of the column $C_{j}^{(g) h}$ (respectively the row $R_{i}^{(g)}$ ), the set of all the obtained blocks provides a PBIP with 7 associated classes.

Theorem 9. The partially balanced incomplete block designs with the parameters:

$$
\begin{gathered}
v=w n l, \quad b=w(w-1) n(n-1)(s+2) C_{s}^{l} / 2, \quad \lambda_{0}=r=(w-1)(n-1) C_{s-1}^{l-1}(s+2), \quad k=2 s, \\
\lambda_{1}=s(n-1)(w-1) C_{s-2}^{l-2}, \quad \lambda_{2}=s(w-1) C_{s-1}^{l-1}, \quad \lambda_{3}=(w-1)(l-2) C_{s-3}^{l-3}, \quad \lambda_{4}=0, \\
\lambda_{5}=2(n-1) C_{s-2}^{l-2}, \quad \lambda_{6}=2 C_{s-1}^{l-1}, \quad \lambda_{7}=4 C_{s-2}^{l-2}
\end{gathered}
$$

are generalized rectangular right angular $\mathrm{GPBIB}_{7}$ designs.

Proof. The design parameters are deduced from the construction method.

Remark 5. For $w=2$, the $\mathrm{GPBIB}_{7}$ design of Theorem 9 is a rectangular right angular $\mathrm{PBIB}_{7}$ design with parameters as in Proposition 5 of [8].

Proposition 10. Let $\mathrm{SPBIB}_{7}$ be a design with parameters:

$$
\begin{gathered}
v=w n l, \quad b=w(w-1) n(n-1)(s+2) C_{s}^{l} / 2, \quad \lambda_{0}=r=(w-1)(n-1) C_{s-1}^{l-1}(s+2), \quad k=2 s, \\
\lambda_{1}=s(n-1)(w-1) C_{s-2}^{l-2}, \quad \lambda_{2}=s(w-1) C_{s-1}^{l-1}, \quad \lambda_{3}=(w-1)(l-2) C_{s-3}^{l-3}, \quad \lambda_{4}=0, \\
\lambda_{5}=2(n-1) C_{s-2}^{l-2}, \quad \lambda_{6}=2 C_{s-1}^{l-1}, \quad \lambda_{7}=4 C_{s-2}^{l-2}
\end{gathered}
$$

For $n$ or $l$ even and $s$ divisor of $l$ or $s$, the GPBIB $_{7}$ design is a resolvable PBIB designs $\left(R G P B I B_{7}\right)$ with $r$ parallel classes where each parallel classes contain $q=w \frac{n l}{2 s}$ blocks.

Example 4. Let $v=3 \times 3 \times 3$ treatments be arranged in the three following arrays:

\begin{tabular}{lll}
\hline 1 & 2 & 3 \\
4 & 5 & 6 \\
7 & 8 & 9 \\
\hline & & \\
\hline 10 & 11 & 12 \\
13 & 14 & 15 \\
16 & 17 & 18 \\
\hline & & \\
\hline 19 & 20 & 21 \\
22 & 23 & 24 \\
25 & 2 & 27 \\
\hline
\end{tabular}

The construction method for $(s=3)$, give a generalized rectangular right angular $G P B I B_{7}$ design with parameters:

$$
\begin{gathered}
v=27, \quad b=90, \quad r=20, \quad k=6, \quad \lambda_{1}=12, \quad \lambda_{2}=6, \\
\lambda_{3}=4, \quad \lambda_{4}=0, \quad \lambda_{5}=4, \quad \lambda_{6}=2, \quad \lambda_{7}=4
\end{gathered}
$$

To illustrate the method, we applying the construction method for the columns and rows of the first array, where each column represents a block: 


\begin{tabular}{|c|c|c|c|c|c|c|c|c|c|}
\hline$b_{1}$ & $b_{2}$ & $b_{3}$ & $b_{4}$ & $b_{5}$ & $b_{6}$ & $b_{7}$ & $b_{8}$ & $b_{9}$ & $b_{10}$ \\
\hline 1 & 1 & 4 & 2 & 2 & 5 & 3 & 3 & 6 & 1 \\
\hline 11 & 11 & 14 & 10 & 10 & 13 & 10 & 10 & 13 & 20 \\
\hline 12 & 12 & 15 & 12 & 12 & 15 & 11 & 11 & 14 & 21 \\
\hline 4 & 7 & 7 & 5 & 8 & 8 & 6 & 9 & 9 & 4 \\
\hline 14 & 17 & 17 & 13 & 16 & 16 & 13 & 16 & 16 & 23 \\
\hline 15 & 18 & 18 & 15 & 18 & 18 & 14 & 17 & 17 & 24 \\
\hline$b_{11}$ & $b_{12}$ & $b_{13}$ & $b_{14}$ & $b_{15}$ & $b_{16}$ & $b_{17}$ & $b_{18}$ & $b_{19}$ & $b_{20}$ \\
\hline 1 & 4 & 2 & 2 & 5 & 3 & 3 & 6 & 1 & 1 \\
\hline 20 & 23 & 19 & 19 & 22 & 19 & 19 & 22 & 2 & 2 \\
\hline 21 & 24 & 21 & 21 & 24 & 20 & 20 & 23 & 3 & 3 \\
\hline 7 & 7 & 5 & 8 & 8 & 6 & 9 & 9 & 13 & 16 \\
\hline 26 & 26 & 22 & 25 & 25 & 22 & 25 & 25 & 14 & 17 \\
\hline 27 & 27 & 24 & 27 & 27 & 23 & 26 & 26 & 15 & 18 \\
\hline$b_{21}$ & $b_{22}$ & $b_{23}$ & $b_{24}$ & $b_{25}$ & $b_{26}$ & $b_{27}$ & $b_{28}$ & $b_{29}$ & $b_{30}$ \\
\hline 4 & 4 & 7 & 7 & 1 & 1 & 4 & 4 & 7 & 7 \\
\hline 5 & 5 & 8 & 8 & 2 & 2 & 5 & 5 & 8 & 8 \\
\hline 6 & 6 & 9 & 9 & 3 & 3 & 6 & 6 & 9 & 9 \\
\hline 10 & 16 & 10 & 13 & 22 & 25 & 19 & 25 & 19 & 22 \\
\hline 11 & 17 & 11 & 14 & 23 & 26 & 20 & 26 & 20 & 23 \\
\hline 12 & 18 & 12 & 15 & 24 & 27 & 21 & 27 & 21 & 24 \\
\hline
\end{tabular}

\section{Construction of the U-Type Designs Based on Resolvable GPBIB $m$ Designs ( $m=4,5$ and 7$)$}

In this section we apply the RBIBD method (see [6]) on our resolvable rectangular right angular RGPBIB $_{m}$ designs ( $m=4,5$ and 7) to obtain a series of $U$-type designs $U\left(v ; q^{r}\right)$.

Let a resolvable GPBIB $m$ designs ( $m=4,5$ and 7) with $r$ parallel classes $\mathrm{PC}_{1}, \cdots, \mathrm{PC}_{r}$ where each $j$-th class contains $q=b / r$ blocks $(1 \leq j \leq r)$. Then we can construct a $U$-type design $U\left(v ; q^{r}\right)$ from resolvable $G P B I B_{m}$ designs ( $m=4,5$ and 7$)$ as follows:

\section{Algorithm RGPBIB $B_{m}-U D$}

- Step 1. Give a natural order $1, \cdots, q$ to the $q$ blocks in each parallel class $\mathrm{PC}_{j}, j=1, \cdots, r$.

- Step 2. For each $\mathrm{PC}_{j}$, construct a $q$-level column $x^{j}=\left(x_{\alpha j}\right)$ as follows: Set $x_{\alpha j}=u$, if treatment $\alpha$ is contained in the $u$-th block of $\mathrm{PC}_{j}, u=1,2, \cdots, q$

- Step 3. The $r q$-level columns constructed from $\mathrm{PC}_{j}, j=1, \cdots, r$ form a $U\left(v ; q^{r}\right)$.

Proposition 11. For $v=$ wnl runs $(w \geq 2, n \geq 2, l \geq 2)$, a series of $U$-type $U\left(v ; q^{r}\right)$ designs exist:

- $U\left(w n l ; \frac{w n l^{(n-1) C_{s-1}^{l-1}}}{2 s}\right), n$ or $l$ even and $s$ divisor of $n$ or $l$.

- $U\left(w n l ; \frac{w n l^{s(w-1) n(n-1) C_{s-1}^{l}}}{2 s}\right), n$ or $l$ even and $s$ divisor of $n$ or $l$. 
- $\quad U\left(w n l ; \frac{w n l^{s(w-1)(n-1) C_{s-1}^{l}}}{2 s}\right), n$ or $l$ even and $s$ divisor of $n$ or $l$.

- $U\left(w n l ; \frac{n l^{(n-1) C_{s-1}^{l-1}}}{2 s}\right), n$ or $l$ even and $s$ divisor of $n$ or $l$.

- $U\left(w n l ; \frac{w n l^{(s+2)(w-1)(n-1) C_{s-1}^{l-1}}}{2 s}\right), n$ or $l$ even and $s$ divisor of $n$ or $l$.

Proof. applying the $R G P B I B_{m}-U D$ Algorithm on each resolvable rectangular right angular $G P B I B_{m}(m=4,5$ and 7) of the Proposition 1, 5.

Example 5. Applying the RGPBIB ${ }_{m}-U D$ Algorithm on the resolvable rectangular right angular $G P B I B_{7}$ of Example 1, we obtain the following $U$-type $U\left(48,4^{9}\right)$ with 48 runs and nine 4-level factors.

\begin{tabular}{|c|c|c|c|c|c|c|c|c|c|}
\hline Runs & Factor $_{1}$ & Factor $_{2}$ & Factor $_{3}$ & Factor $_{4}$ & Factor $_{5}$ & Factor $_{6}$ & Factor $_{7}$ & Factor $_{8}$ & Factor $_{9}$ \\
\hline 1 & 1 & 1 & 1 & 1 & 1 & 1 & 1 & 1 & 1 \\
\hline 2 & 1 & 1 & 1 & 3 & 3 & 3 & 3 & 3 & 3 \\
\hline 3 & 3 & 3 & 3 & 1 & 1 & 1 & 3 & 3 & 3 \\
\hline 4 & 3 & 3 & 3 & 3 & 3 & 3 & 1 & 1 & 1 \\
\hline 5 & 1 & 2 & 2 & 1 & 2 & 2 & 1 & 2 & 2 \\
\hline 6 & 1 & 2 & 2 & 3 & 4 & 4 & 3 & 4 & 4 \\
\hline 7 & 3 & 4 & 4 & 1 & 2 & 2 & 3 & 4 & 4 \\
\hline 8 & 3 & 4 & 4 & 3 & 4 & 4 & 1 & 2 & 2 \\
\hline 9 & 2 & 1 & 2 & 2 & 1 & 2 & 2 & 1 & 2 \\
\hline 10 & 2 & 1 & 2 & 4 & 3 & 4 & 4 & 3 & 4 \\
\hline 11 & 4 & 3 & 4 & 2 & 3 & 4 & 4 & 3 & 4 \\
\hline 12 & 4 & 3 & 4 & 4 & 3 & 4 & 2 & 1 & 2 \\
\hline 13 & 2 & 2 & 1 & 2 & 2 & 1 & 2 & 2 & 1 \\
\hline 14 & 2 & 2 & 1 & 4 & 4 & 3 & 4 & 4 & 3 \\
\hline 15 & 4 & 4 & 3 & 2 & 2 & 1 & 4 & 4 & 3 \\
\hline 16 & 4 & 4 & 3 & 4 & 4 & 3 & 2 & 2 & 1 \\
\hline 17 & 1 & 1 & 1 & 1 & 1 & 1 & 1 & 1 & 1 \\
\hline 18 & 1 & 1 & 1 & 3 & 3 & 3 & 3 & 3 & 3 \\
\hline 19 & 3 & 3 & 3 & 1 & 1 & 1 & 3 & 3 & 3 \\
\hline 20 & 3 & 3 & 3 & 3 & 3 & 3 & 1 & 1 & 1 \\
\hline 21 & 1 & 2 & 2 & 1 & 2 & 2 & 1 & 2 & 2 \\
\hline 22 & 1 & 2 & 2 & 3 & 4 & 4 & 3 & 4 & 4 \\
\hline 23 & 3 & 4 & 4 & 1 & 2 & 2 & 3 & 4 & 4 \\
\hline 24 & 3 & 4 & 4 & 3 & 4 & 4 & 1 & 2 & 2 \\
\hline 25 & 2 & 1 & 2 & 2 & 1 & 2 & 2 & 1 & 2 \\
\hline 26 & 2 & 1 & 2 & 4 & 3 & 4 & 4 & 3 & 4 \\
\hline
\end{tabular}




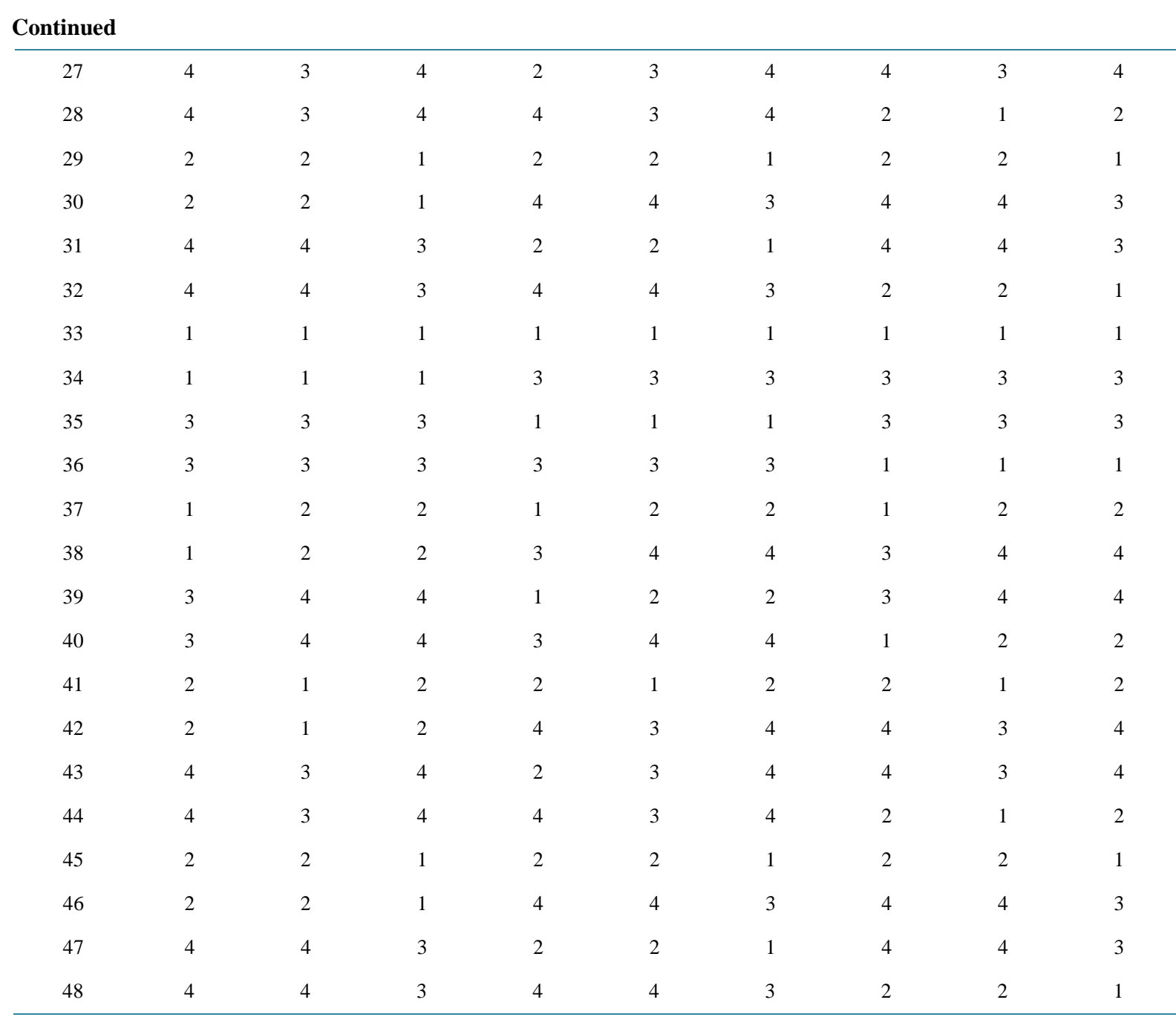

\section{Conclusions}

New association schemes with $m=4,5$ and 7 associated classes called generalized rectangular right angular association schemes for $v=w n l$ treatments arranged in $w \geq 2(n \times l)$ arrays were described and their parameters expressions were given exactly and directly. Some construction methods of PBIB designs based on these association schemes accommodated by accessible method called the Combinatory Method (s) which facilitates the construction application were explained. Moreover, a series of $U$-type designs $U\left(w n l ; \frac{w n l}{2 s}\right)$, by applying the Fang $R B I B D$ method on resolvable generalized rectangular right angular $G P B I B_{m}$ designs $(m=4,5$ and 7$)$ was constructed.

We note that all the construction methods described in this article were programmed with the R-package "CombinS" [9] (the ameliorated version).

\section{References}

[1] Fang, K.T., Ge, G.N., Liu, M. and Qin, H. (2004) Construction of Uniform Designs via Super-Simple Resolvable t-Designs. Utilitas Mathematica, 66, 15-32.

[2] Fang, K.T., Tang, Y. and Yin, J.X. (2005) Resolvable Partially Pairwise Balanced Designs and Their Applications in Computer Experiments. Utilitas Mathematica, 70, 141-157.

[3] Benmatti, A. (1983) Un schéma d'association partiellement équilibré appliqué aux croisements dialléles. Magister 
Thesis. http://biblio.cca-paris.com/index.php?lvl=author_see\&id=1181

[4] Bailey, R.A. (2004) Association Schemes: Designed Experiments, Algebra and Combinatorics. Cambridge University Press, Cambridge. www.cambridge.org/9780521824460

[5] Rezgui, I. and Gheribi-Aoulmi, Z. (2014) New Construction Method of Rectangular PBIB Designs and Singular Group Divisible Designs. Journal of Mathematics and Statistics, 10, 45-48. http://dx.doi.org/10.3844/jmssp.2014.45.48

[6] Fang, K.T., Li, R. and Sudjianto, A. (2006) Design and Modeling for Computer Experiments. Taylor \& Francis Group, LLC, London.

[7] Bose, R.C. and Nair, K.R. (1939) Partially Balanced Incomplete Block Designs. Sankhya, 4, 337-372.

[8] Rezgui, I., Gheribi-Aoulmi, Z. and Monod, H. (2013) New Association Schemes with 4, 5 and 7 Associate Classes and Their Associated Partially Balanced Incomplete Block Designs. Advances and Applications in Discrete Mathematics, 12, 207-215.

[9] Laib, M., Rezgui, I., Gheribi-Aoulmi, Z. and Monod, H. (2013) Package “CombinS”: Constructions Method of Rectangular PBIB and Rectangular Right Angular PBIB(m) $(m=4,5$ and 7) Designs. Version 1.0. http://cran.r-project.org/web/packages/CombinS/CombinS.pdf 
Scientific Research Publishing (SCIRP) is one of the largest Open Access journal publishers. It is currently publishing more than 200 open access, online, peer-reviewed journals covering a wide range of academic disciplines. SCIRP serves the worldwide academic communities and contributes to the progress and application of science with its publication.

Other selected journals from SCIRP are listed as below. Submit your manuscript to us via either submit@scirp.org or Online Submission Portal.
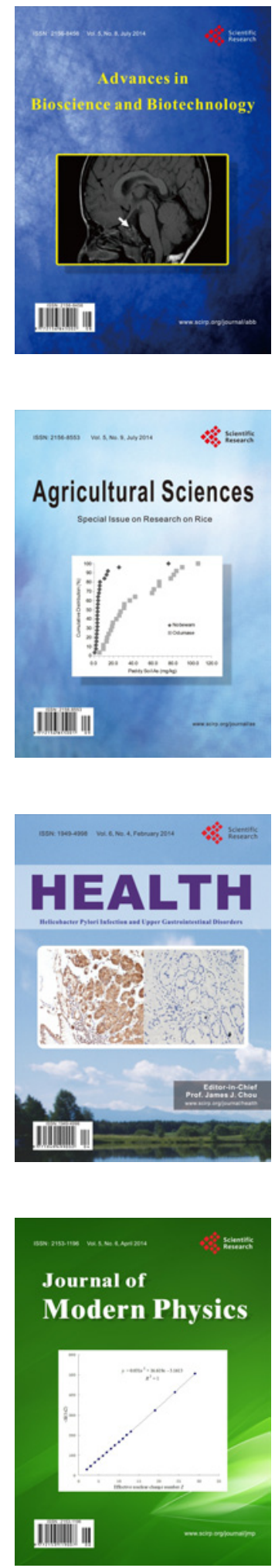
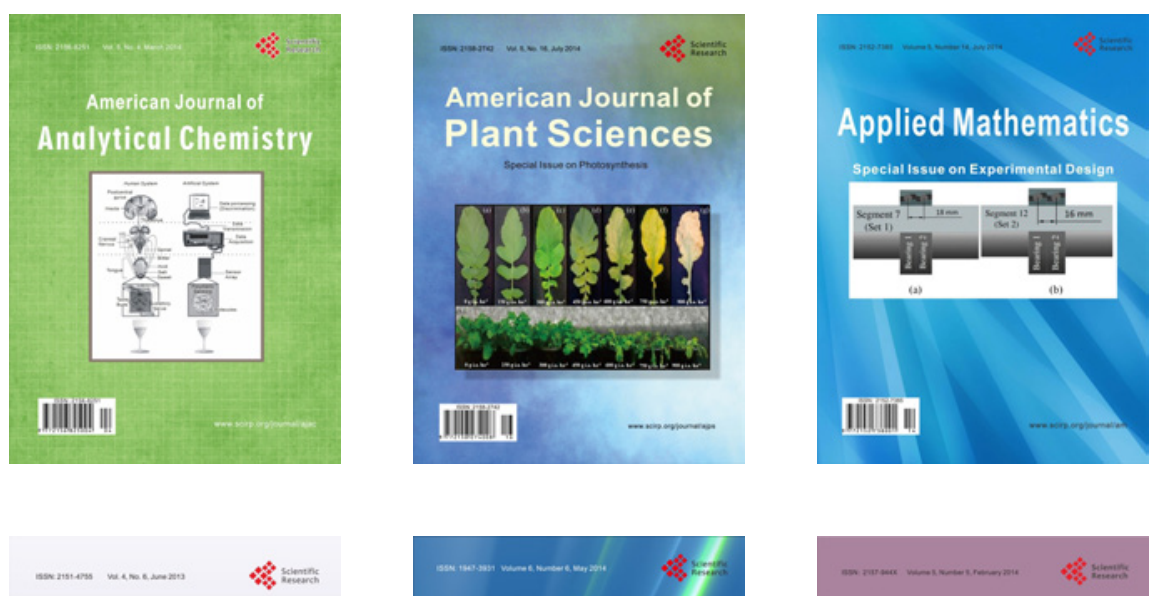

Creative Education
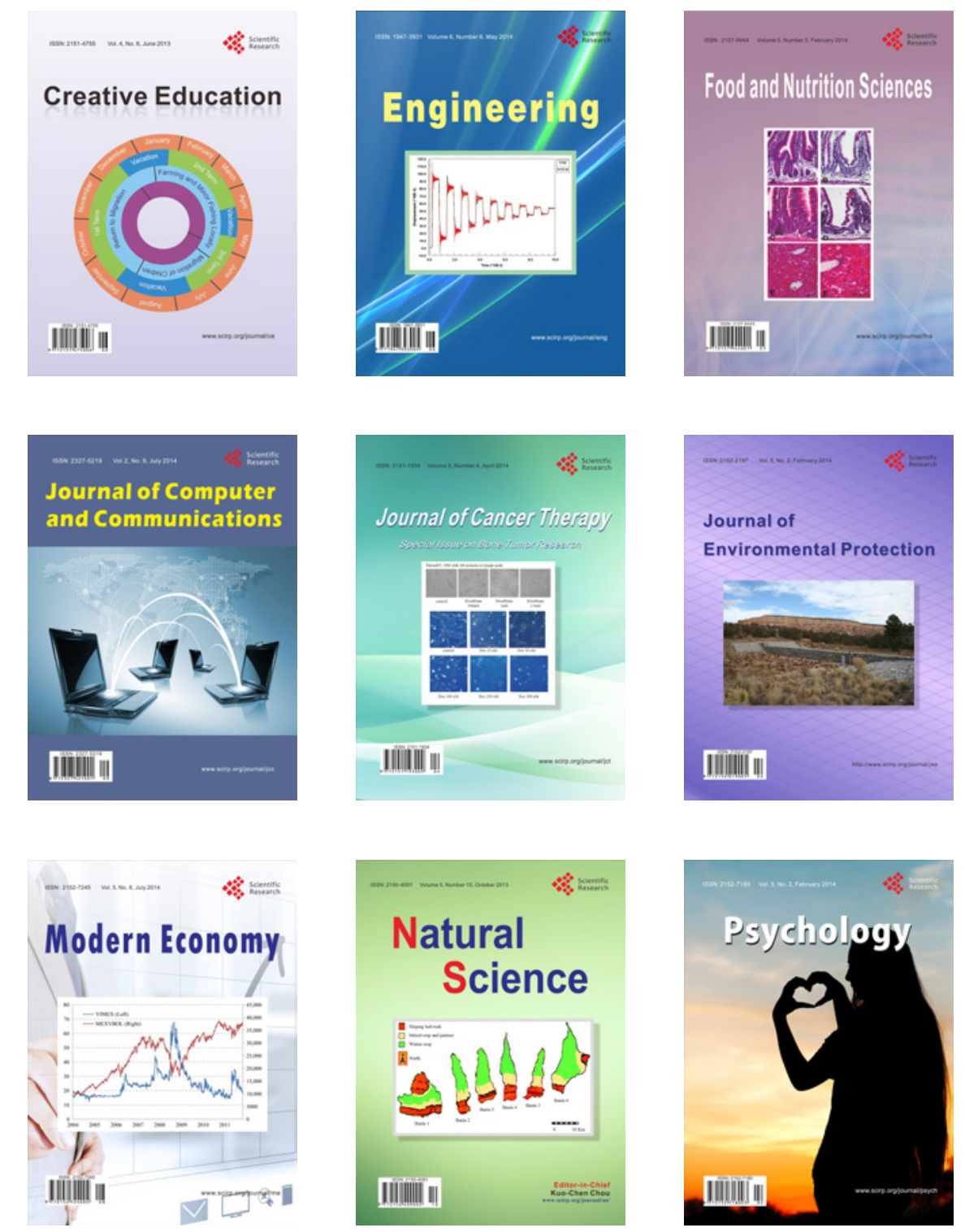